\title{
The Tippedisk: A Minimal Model For Friction-Induced Inversion
}

\author{
Simon Sailer, Simon R. Eugster, Remco I. Leine
}

\author{
Institute for Nonlinear Mechanics \\ University of Stuttgart \\ Pfaffenwaldring 9, 70569, Germany \\ [sailer, eugster, leine]@inm.uni-stuttgart.de
}

\begin{abstract}
The tippedisk is a new mechanical-mathematical archetype for friction induced instability phenomena, showing an inversion similar to the inversion of the tippetop. Unlike the tippetop, the tippedisk has no rotational symmetry, which greatly complicates its analysis. Since the system cannot be reduced to a planar one, one has to consider the full three-dimensional kinematics, being intrinsically nonlinear. In this work a new minimal model is derived that contains the main relevant physical effects so that the inversion phenomenon can be described qualitatively. The in-depth analysis leads to slow-fast systems with homoclinic connections and global bifurcations.
\end{abstract}

Keywords: gyroscopic system, friction-induced instability, dynamics, tippetop.

\section{INTRODUCTION}

Various gyroscopic systems which are interacting with a horizontal frictional support, such as the Euler disk [1, 2], the rattleback [3, 4] and the tippetop [5, 6, 7, 8], form a scientific playground for research in theoretical mechanics. The tippetop [5, 6], as well as the related dynamics of spinning eggs $[9,10]$, correspond to a subclass of gyroscopic systems which show inversion phenomena. The tippetop is a rotationally symmetric top, consisting of a spherical body and a stem attached to it. The center of gravity (COG) does not coincide with the geometric center, such that the stem remains pointing upwards as the top rotates slowly in its non-inverted orientation. If the top is spun fastly around its axis of symmetry, gravitational, normal and friction forces are acting on the top, such that the top starts to invert its orientation and balances on its stem. This phenomenon of inversion also occurs for other axisymmetric bodies with rotational symmetry in inertia and geometry, for example spinning eggs $[9,10,11]$. But what happens if this symmetry does not exist? In [12], we introduced the "tippedisk" as new archetype of a three-dimensional rigid body system with frictional contact. The tippedisk can be seen as a thin disk for which the COG does not coincide with the geometric center. If the tippedisk is spun rapidly around an in-plane axis, one can observe that the COG rises until the disk remains in an inverted configuration, see Fig. 1. The inversion phenomenon is therefore not restricted to axisymmetric rigid bodies and also takes place for the tippedisk. In this work, we derive a minimal model able to describe the inversion of the tippedisk. A suitable parametrization is introduced, which is able to describe the inversion phenomenon. The model developed in this work forms the basis for in-depth nonlinear analysis of the dynamics of the tippedisk, e.g., $[13,14]$.

\section{MECHANICAL MODEL}

The mechanical system depicted in Fig. 2 consists of an unbalanced rigid disk with mass $m$, radius $r$, eccentricity $e$, thickness $h$ and a flat frictional support. Since we are only interested in the essential physical phenomena, the contact kinematics is simplified by assuming the disk to be infinitely thin. According to this approximation, the contact point $C_{1}$, i.e., the point with minimal height, lies on a circle around the geometric center $G$, in the inclined $\Pi$-plane. The vertical projection of the contact point $C_{1}$ onto the horizontal support is denoted by $D_{1}$. Both the disk and the flat support are considered to be perfectly rigid, so that penetration is not possible. We introduce an orthonormal 

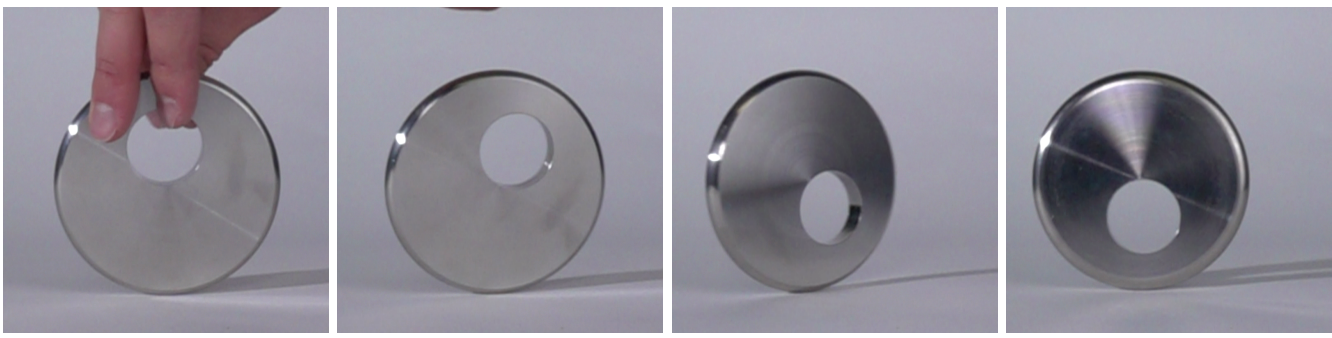

Figure 1: Stroboscopic image sequence showing the inversion phenomenon of the tippedisk. First Picture: Non-inverted configuration; Last Picture: Tippedisk spinning in its inverted configuration.

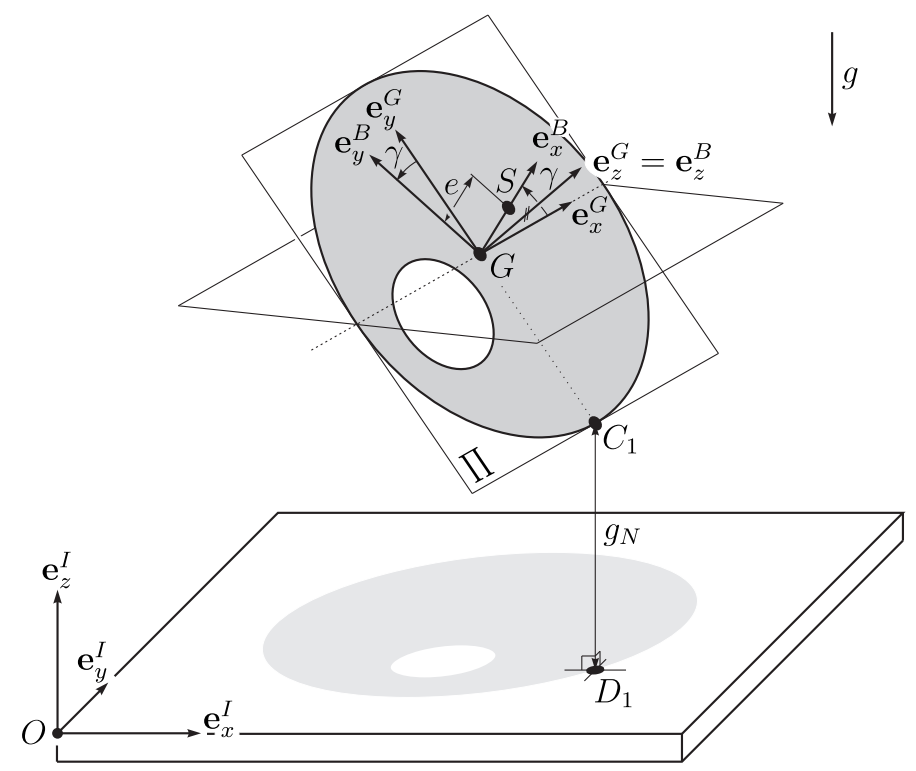

Figure 2: Mechanical model of the tippedisk, showing the definition of the floating $G$-frame and the contact points $C_{1}$ and $D_{1}$.

inertial frame $I=\left(O, \mathbf{e}_{x}^{I}, \mathbf{e}_{y}^{I}, \mathbf{e}_{z}^{I}\right)$ attached to the origin $O$, where $\mathbf{e}_{z}^{I}$ is normal to the flat support. The right-handed body-fixed $B$-frame $B=\left(G, \mathbf{e}_{x}^{B}, \mathbf{e}_{y}^{B}, \mathbf{e}_{z}^{B}\right)$ is attached to the geometric center $G$ of the disk, such that $\mathbf{e}_{z}^{B}$ is normal to the surface of the disk. The axis $\mathbf{e}_{x}^{B}$ is defined as the normalized vector of $\mathbf{r}_{G S}$, which points from the geometric center $G$ to the center of gravity $S$. The inertia tensor with respect to $G$ expressed in the body-fixed $B$-frame is given as ${ }_{B} \Theta_{G}=\operatorname{diag}(A, B, C)$, where $B<A<C$ holds. To describe the kinematics of the point $C_{1}$, we introduce analogue to [15] a floating coordinate system $G=\left(G, \mathbf{e}_{x}^{G}, \mathbf{e}_{y}^{G}, \mathbf{e}_{z}^{G}\right)$, which is attached to the geometric center $G$. Its unit vector $\mathbf{e}_{z}^{G}$ corresponds to $\mathbf{e}_{z}^{B}$. Since the cross product $\mathbf{e}_{z}^{I} \times \mathbf{e}_{z}^{B}$ is perpendicular to $\mathbf{e}_{z}^{I}$ and $\mathbf{e}_{z}^{B}$, we define the orthogonal vector

$$
\mathbf{e}_{x}^{G}:=\frac{\mathbf{e}_{z}^{I} \times \mathbf{e}_{z}^{B}}{\left\|\mathbf{e}_{z}^{I} \times \mathbf{e}_{z}^{B}\right\|} \quad \text { with } \quad\left\|\mathbf{e}_{z}^{I} \times \mathbf{e}_{z}^{B}\right\|=\sqrt{1-R_{33}^{2}},
$$

as the horizontal unit vector of the floating coordinate system, where $R_{33}$ denoted the third diagonal element of the rotation matrix $\mathbf{R}$ given below. The symbol $\| .||$ denotes the Euclidean norm. Definition (1) is only valid for non-horizontal configurations with $R_{33} \neq 1$. For horizontal configurations, note that $\mathbf{e}_{x}^{G}$ can not be determined uniquely. If $R_{33} \neq 1$, we can obtain with

$$
\mathbf{e}_{y}^{G}:=\mathbf{e}_{z}^{B} \times \mathbf{e}_{x}^{G}
$$

a right handed orthonormal frame, such that the floating coordinate frame $G=\left(G, \mathbf{e}_{x}^{G}, \mathbf{e}_{y}^{g}, \mathbf{e}_{z}^{G}\right)$ is fully defined. The point $C_{1}$ with minimal height can be described with respect to the geometric 


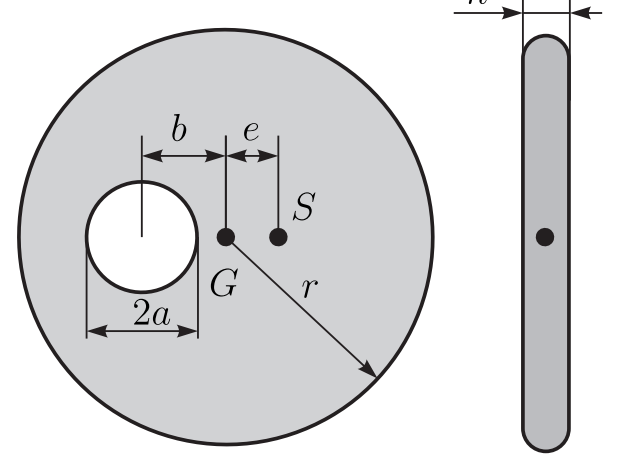

Figure 3: Dimensions of the tippedisk.

center $G$ as

$$
\mathbf{r}_{G C_{1}}=-r \mathbf{e}_{y}^{G} .
$$

The distance between the contact point $C_{1}$ and the flat support defines the signed gap $g_{N}$

$$
g_{N}=\mathbf{r}_{O C_{1}} \cdot \mathbf{e}_{z}^{I}=\left(\mathbf{r}_{O G}+\mathbf{r}_{G C_{1}}\right) \cdot \mathbf{e}_{z}^{I},
$$

which is equal to the projection of $\mathbf{r}_{O C_{1}}$ onto the $\mathbf{e}_{z}^{I}$-axis.

\section{DIMENSIONS}

In the following we consider a stainless steel disk, which is depicted in Fig. 3. The dimensions and mass properties are given in Table 1, for a detailed derivation of the principal moments of inertia, we refer to [12].

Table 1: Dimensions and mass properties of the tippedisk.

\begin{tabular}{|c|c|c|c|}
\hline Property & Parameter & Magnitude & Unit \\
\hline Disk radius & $r$ & 0.045 & $\mathrm{~m}$ \\
\hline Hole radius & $a$ & 0.015 & $\mathrm{~m}$ \\
\hline Distance & $b$ & 0.02 & $\mathrm{~m}$ \\
\hline Disk thickness & $h$ & 0.01 & $\mathrm{~m}$ \\
\hline Eccentricity & $e$ & $2.5 \cdot 10^{-3}$ & $\mathrm{~m}$ \\
\hline Mass & $m$ & 0.435 & $\mathrm{~kg}$ \\
\hline${ }_{B} \boldsymbol{\Theta}_{G}(1,1)$ & $A$ & $0.249 \cdot 10^{-3}$ & $\mathrm{~kg} \mathrm{~m}$ \\
\hline${ }_{B} \Theta_{G}(2,2)$ & $B$ & $0.227 \cdot 10^{-3}$ & $\mathrm{~kg} \mathrm{~m}^{2}$ \\
\hline${ }_{B} \Theta_{G}(3,3)$ & $C$ & $0.468 \cdot 10^{-3}$ & $\mathrm{~kg} \mathrm{~m}^{2}$ \\
\hline
\end{tabular}

\section{KINEMATICS}

We parametrize the orientation of the tippedisk using Euler angles $\boldsymbol{\varphi}=[\alpha, \beta, \gamma]^{\mathrm{T}}$. Therefore, the rotating $R$-frame with $\mathbf{e}_{z}^{R}=\mathbf{e}_{z}^{I}, \mathbf{e}_{x}^{R}=\cos \alpha \mathbf{e}_{x}^{I}+\sin \alpha \mathbf{e}_{y}^{I}$ and $\mathbf{e}_{y}^{R}=\mathbf{e}_{z}^{R} \times \mathbf{e}_{x}^{R}$ is introduced. The sequence of rotation is then given as the first rotation with angle $\alpha$ around the $\mathbf{e}_{z}^{I}$-axis, the second rotation with angle $\beta$ around the $\mathbf{e}_{x}^{R}$-axis and the third rotation with angle $\gamma$ around the $\mathbf{e}_{z}^{G}$-axis 
with corresponding elemental rotations

$$
\mathbf{A}_{I R}=\left[\begin{array}{ccc}
\mathrm{c} \alpha & -\mathrm{s} \alpha & 0 \\
\mathrm{~s} \alpha & \mathrm{c} \alpha & 0 \\
0 & 0 & 1
\end{array}\right], \mathbf{A}_{R G}=\left[\begin{array}{ccc}
1 & 0 & 0 \\
0 & \mathrm{c} \beta & -\mathrm{s} \beta \\
0 & \mathrm{~s} \beta & \mathrm{c} \beta
\end{array}\right] \text { and } \mathbf{A}_{G B}=\left[\begin{array}{ccc}
\mathrm{c} \gamma & -\mathrm{s} \gamma & 0 \\
\mathrm{~s} \gamma & \mathrm{c} \gamma & 0 \\
0 & 0 & 1
\end{array}\right],
$$

where the abbreviations $\operatorname{s} \alpha=\sin (\alpha), c \alpha=\cos (\alpha)$ etc. have been used. The rotation matrix

$$
\mathbf{R}(\boldsymbol{\varphi})=\left[\begin{array}{ccc}
\mathrm{c} \alpha \mathrm{c} \gamma-\mathrm{s} \alpha \mathrm{c} \beta \mathrm{s} \gamma & -\mathrm{c} \alpha \mathrm{s} \gamma-\mathrm{s} \alpha \mathrm{c} \beta \mathrm{c} \gamma & \mathrm{s} \alpha \mathrm{s} \beta \\
\mathrm{s} \alpha \mathrm{c} \gamma+\mathrm{c} \alpha \mathrm{c} \beta \mathrm{s} \gamma & -\mathrm{s} \alpha \mathrm{s} \gamma+\mathrm{c} \alpha \mathrm{c} \beta \mathrm{c} \gamma & -\mathrm{c} \alpha \mathrm{s} \beta \\
\mathrm{s} \beta \mathrm{s} \gamma & \mathrm{s} \beta \mathrm{c} \gamma & \mathrm{c} \beta
\end{array}\right]
$$

which per se fulfills the orthogonality condition $\mathbf{R}^{\mathrm{T}} \mathbf{R}=\mathbf{I}$, then describes the relative orientation of the body-fixed $B$-frame with respect to the inertial $I$-frame. ${ }^{1}$ The angular velocity $\boldsymbol{\Omega}$ of the tippedisk expressed in the body-fixed $B$-frame yields

$$
{ }_{B} \boldsymbol{\Omega}=\dot{\alpha} \mathbf{A}_{R B R}^{\mathrm{T}} \mathbf{e}_{z}^{R}+\dot{\beta} \mathbf{A}_{G B}^{\mathrm{T}} \mathbf{e}_{x}^{G}+\dot{\gamma}_{B} \mathbf{e}_{z}^{B}=\left[\begin{array}{c}
\dot{\alpha} \mathrm{s} \beta \mathrm{s} \gamma+\dot{\beta} \mathrm{c} \gamma \\
\dot{\alpha} \beta \mathrm{c} \gamma-\dot{\beta} \mathrm{s} \gamma \\
\dot{\alpha} \mathrm{c} \beta+\dot{\gamma}
\end{array}\right],
$$

where the transformation matrix $\mathbf{A}_{R B}=\mathbf{A}_{R G} \mathbf{A}_{G B}$ has been used. The transformation

$$
{ }_{R} \boldsymbol{\Omega}=\mathbf{A}_{R B B} \boldsymbol{\Omega}=\left[\begin{array}{c}
\dot{\beta} \\
-\dot{\gamma} \mathrm{s} \beta \\
\dot{\alpha}+\dot{\gamma} \mathrm{c} \beta
\end{array}\right]
$$

yields the angular velocity of the tippedisk expressed in the rotating $R$-frame. The relative angular velocity of the $R$-frame is given with respect to the $I$-frame as ${ }_{R} \boldsymbol{\omega}_{I R}=[0,0, \dot{\alpha}]^{\mathrm{T}}$. Using coordinates $x, y$ and $z$, the position of the geometric center $G$ in the rotating $R$-frame is defined as

$$
{ }_{R} \mathbf{r}_{O G}:=\left[\begin{array}{c}
x \\
y \\
z
\end{array}\right] .
$$

The corresponding velocity

$$
R_{\mathbf{v}_{G}}=\left[\begin{array}{c}
\dot{x} \\
\dot{y} \\
\dot{z}
\end{array}\right]+\left[\begin{array}{c}
0 \\
0 \\
\dot{\alpha}
\end{array}\right] \times\left[\begin{array}{c}
x \\
y \\
z
\end{array}\right]=\left[\begin{array}{c}
\dot{x}-y \dot{\alpha} \\
\dot{y}+x \dot{\alpha} \\
\dot{z}
\end{array}\right]
$$

is obtained, using Euler's rule of differentiation ${ }_{R} \mathbf{v}_{G}=\left({ }_{R} \mathbf{r}_{O G}\right)^{\cdot}+{ }_{R} \boldsymbol{\omega}_{I R} \times{ }_{R} \mathbf{r}_{O G}$. Introducing the set of coordinates $\mathbf{q}=[x, y, z, \alpha, \beta, \gamma]^{\mathrm{T}} \in \mathbb{R}^{6}$, the Jacobian matrices of rotation $\mathbf{J}_{R}$ and translation $\mathbf{J}_{G}$ are obtained with (7) and (10) as

$$
{ }_{B} \mathbf{J}_{R}=\frac{\partial_{B} \boldsymbol{\Omega}}{\partial \dot{\mathbf{q}}}=\left[\begin{array}{cccccc}
0 & 0 & 0 & \mathrm{~s} \beta \mathrm{s} \gamma & \mathrm{c} \gamma & 0 \\
0 & 0 & 0 & \mathrm{~s} \beta \mathrm{c} \gamma & -\mathrm{s} \gamma & 0 \\
0 & 0 & 0 & \mathrm{c} \beta & 0 & 1
\end{array}\right],{ }_{R} \mathbf{J}_{G}=\frac{\partial_{R} \mathbf{v}_{G}}{\partial \dot{\mathbf{q}}}=\left[\begin{array}{rrrrrr}
1 & 0 & 0 & -y & 0 & 0 \\
0 & 1 & 0 & +x & 0 & 0 \\
0 & 0 & 1 & 0 & 0 & 0
\end{array}\right] .
$$

The relative position of the center of gravity $S$ with respect to the geometric center $G$ is given as $\mathbf{r}_{G S}=e \mathbf{e}_{x}^{B}$, using the eccentric distance $e$. For reasons of notation, the bijective map

$$
j: \quad \mathbb{R}^{3} \rightarrow \mathbb{R}^{3 \times 3}: \quad \mathbf{a}=\left[\begin{array}{l}
a_{1} \\
a_{2} \\
a_{3}
\end{array}\right] \mapsto \tilde{\mathbf{a}}=\left[\begin{array}{rrr}
0 & -a_{3} & a_{2} \\
a_{3} & 0 & -a_{1} \\
-a_{2} & a_{1} & 0
\end{array}\right],
$$

is introduced, such that the cross product $\mathbf{a} \times \mathbf{b}$ can be written as matrix product of $\tilde{\mathbf{a}} \mathbf{b}$.

\footnotetext{
${ }^{1}$ The rotation matrix $\mathbf{R}$ becomes singular for $\beta=0$ and $\beta=\pi$. However, the following simulation results show that the inversion of the tippedisk is far from singularity, which proves the validity of this parameterization.
} 


\section{KINETICS}

The virtual work $[16,12]$ of a single rigid body is given coordinate free as

$$
\delta W=\left[\begin{array}{c}
\delta \mathbf{r}_{G} \\
\delta \boldsymbol{\varphi}
\end{array}\right] \cdot\left(\left[\begin{array}{cc}
m \mathbb{1} & m \tilde{\mathbf{r}}_{G S}^{\mathrm{T}} \\
m \tilde{\mathbf{r}}_{G S} & \boldsymbol{\Theta}_{G}
\end{array}\right]\left[\begin{array}{c}
\mathbf{a}_{G} \\
\boldsymbol{\Psi}
\end{array}\right]-\left[\begin{array}{c}
-m \boldsymbol{\Omega} \times\left(\boldsymbol{\Omega} \times \mathbf{r}_{G S}\right) \\
-\boldsymbol{\Omega} \times\left(\boldsymbol{\Theta}_{G} \boldsymbol{\Omega}\right)
\end{array}\right]-\left[\begin{array}{c}
\mathbf{F}_{G}^{\mathrm{ext}} \\
\mathbf{M}_{G}^{\mathrm{ext}}
\end{array}\right]\right) .
$$

According to Eq. (9) the geometric center $G$ is parameterized with respect to the rotating $R$-frame ${ }^{2}$. Since the inertia tensor in the body-fixed $B$-frame ${ }_{B} \boldsymbol{\Theta}_{G}$ is constant, it is convenient to express the angular velocity $\boldsymbol{\Omega}$ and the angular acceleration $\boldsymbol{\Psi}$ with respect to the body-fixed $B$-frame, so that the virtual work Eq. (13) expressed in mixed $R$ - and $B$-coordinates reads as

$$
\delta W=\left[\begin{array}{c}
{ }_{R} \boldsymbol{\delta} \mathbf{r}_{G} \\
{ }_{B} \boldsymbol{\delta} \boldsymbol{\varphi}
\end{array}\right]^{\mathrm{T}}\left(\left[\begin{array}{cc}
m \mathbf{I} & m_{R} \tilde{\mathbf{r}}_{G S}^{\mathrm{T}} \mathbf{A}_{R B} \\
m_{B} \tilde{\mathbf{r}}_{G S} \mathbf{A}_{R B}^{\mathrm{T}} & { }_{B} \boldsymbol{\Theta}_{G}
\end{array}\right]\left[\begin{array}{c}
{ }_{R} \mathbf{a}_{G} \\
{ }_{B} \boldsymbol{\Psi}
\end{array}\right]-\left[\begin{array}{c}
-m_{R} \boldsymbol{\Omega} \times\left({ }_{R} \boldsymbol{\Omega} \times{ }_{R} \mathbf{r}_{G S}\right) \\
-{ }_{B} \boldsymbol{\Omega} \times\left({ }_{B} \boldsymbol{\Theta}_{G B} \boldsymbol{\Omega}\right)
\end{array}\right]\right)-\delta W^{\text {ext }},
$$

where

$$
\delta W^{\mathrm{ext}}=\delta \mathbf{q}^{\mathrm{T}} \mathbf{f}^{\mathrm{ext}}=\left[\begin{array}{c}
{ }_{R} \delta \mathbf{r}_{G} \\
{ }_{B} \delta \boldsymbol{\varphi}
\end{array}\right]^{\mathrm{T}}\left[\begin{array}{c}
{ }_{R} \mathbf{F}_{G}^{\mathrm{ext}} \\
{ }_{B} \mathbf{M}_{G}^{\mathrm{ext}}
\end{array}\right]
$$

denotes the virtual work of external forces an torques caused by force elements or contact forces. The introduced kinematics from Eq. (9), induces the variation of the geometric center as

$$
{ }_{R} \delta \mathbf{r}_{G}={ }_{R} \mathbf{J}_{G} \delta \mathbf{q} .
$$

Together with the $\delta \mathbf{q}$-induced variation of the orientation

$$
{ }_{B} \delta \boldsymbol{\varphi}={ }_{B} \mathbf{J}_{R} \delta \mathbf{q},
$$

the virtual work (induced by the variation of coordinates $\delta \mathbf{q}$ ) yields

$$
\delta W=\delta \mathbf{q}^{\mathrm{T}}\left[\begin{array}{c}
{ }_{R} \mathbf{J}_{G} \\
{ }_{B} \mathbf{J}_{R}
\end{array}\right]^{\mathrm{T}} \cdot\left(\left[\begin{array}{cc}
m \mathbf{I} & m_{R} \tilde{\mathbf{r}}_{G S}^{\mathrm{T}} \mathbf{A}_{R B} \\
m_{B} \tilde{\mathbf{r}}_{G S} \mathbf{A}_{R B}^{\mathrm{T}} & { }_{B} \boldsymbol{\Theta}_{G}
\end{array}\right]\left[\begin{array}{c}
{ }_{R} \mathbf{a}_{G} \\
{ }_{B} \boldsymbol{\Psi}
\end{array}\right]-\left[\begin{array}{c}
-m_{R} \boldsymbol{\Omega} \times\left({ }_{R} \boldsymbol{\Omega} \times{ }_{R} \mathbf{r}_{G S}\right) \\
-{ }_{B} \boldsymbol{\Omega} \times\left({ }_{B} \boldsymbol{\Theta}_{G B} \boldsymbol{\Omega}\right)
\end{array}\right]\right)-\delta W^{\mathrm{ext}},
$$

from which the equation of motion can be extracted as

$$
\left[\begin{array}{c}
{ }_{R} \mathbf{J}_{G} \\
{ }_{B} \mathbf{J}_{R}
\end{array}\right]^{\mathrm{T}} \cdot\left(\left[\begin{array}{cc}
m \mathbf{I} & m_{R} \tilde{\mathbf{r}}_{G S}^{\mathrm{T}} \mathbf{A}_{R B} \\
m_{B} \tilde{\mathbf{r}}_{G S} \mathbf{A}_{R B}^{\mathrm{T}} & { }_{B} \boldsymbol{\Theta}_{G}
\end{array}\right]\left[\begin{array}{c}
{ }_{R} \mathbf{a}_{G} \\
{ }_{B} \boldsymbol{\Psi}
\end{array}\right]-\left[\begin{array}{c}
-m_{R} \boldsymbol{\Omega} \times\left({ }_{R} \boldsymbol{\Omega} \times{ }_{R} \mathbf{r}_{G S}\right) \\
-{ }_{B} \boldsymbol{\Omega} \times\left({ }_{B} \boldsymbol{\Theta}_{G B} \boldsymbol{\Omega}\right)
\end{array}\right]\right)=\mathbf{f}^{\mathbf{e x t}} .
$$

Inserting the introduced kinematics in Eq. (19) yields an equation of the form

$$
\mathbf{M}(\mathbf{q}) \ddot{\mathbf{q}}-\mathbf{h}(\mathbf{q}, \dot{\mathbf{q}})=\mathbf{f}^{\mathrm{ext}}
$$

with symmetric mass matrix

$$
\begin{gathered}
\mathbf{M}(\mathbf{q})=\left[\begin{array}{ll}
\mathbf{M}_{11} & \mathbf{M}_{12} \\
\text { sym. } & \mathbf{M}_{22}
\end{array}\right], \\
\mathbf{M}_{11}=\left[\begin{array}{ccc}
m & 0 & 0 \\
0 & m & 0 \\
0 & 0 & m
\end{array}\right], \\
\mathbf{M}_{22}=\left[\begin{array}{ccc}
M_{22,11} & \cdot & \mathrm{sym} . \\
M_{22,12} & A \mathrm{c}^{2} \gamma+B \mathrm{~s}^{2} \gamma & \cdot \\
M_{22,13} & 0 & C
\end{array}\right], \\
\mathbf{M}_{12}=\left[\begin{array}{ccc}
-m y-m e c \beta \mathrm{s} \gamma & 0 & -m e s \gamma \\
m x+m e c \gamma & -m e s \beta \mathrm{s} \gamma & m e c \beta \mathrm{c} \gamma \\
0 & m e c \beta \mathrm{s} \gamma & m e s \beta \mathrm{c} \gamma
\end{array}\right],
\end{gathered}
$$

\footnotetext{
${ }^{2}$ In [12] there is a typo in Eq. (2.36), since with ${ }_{I} \mathbf{a}_{P}$ the acceleration of the geometric center must correspond to ${ }_{I} \mathbf{a}_{G}$.
} 


$$
\begin{aligned}
& M_{22,11}=m\left(x^{2}+y^{2}\right)+2 m e(x \mathrm{c} \gamma+y \mathrm{c} \beta \mathrm{s} \gamma)+\left(A \mathrm{~s}^{2} \gamma+B \mathrm{c}^{2} \gamma\right) \mathrm{s}^{2} \beta+C \mathrm{c}^{2} \beta, \\
& M_{22,12}=(A-B) \mathrm{s} \beta \mathrm{s} \gamma \mathrm{c} \gamma-m e x \mathrm{~s} \beta \mathrm{s} \gamma, \\
& M_{22,13}=m e x c \beta \mathrm{c} \gamma+m e y \mathrm{~s} \gamma+C \mathrm{c} \beta,
\end{aligned}
$$

and vector of gyroscopic forces

$$
\begin{aligned}
& \mathbf{h}(\mathbf{q}, \dot{\mathbf{q}}):=\left[\begin{array}{llllll}
h_{1} & h_{2} & h_{3} & h_{4} & h_{5} & h_{6}
\end{array}\right]^{\mathrm{T}}, \\
& h_{1}=m\left[x \dot{\alpha}^{2}+2 \dot{y} \dot{\alpha}\right]+m e\left[\left(\dot{\alpha}^{2}+\dot{\gamma}^{2}\right) \mathrm{c} \gamma-2 \dot{\alpha} \dot{\beta} \mathrm{s} \beta \mathrm{s} \gamma+2 \dot{\alpha} \dot{\gamma} \mathrm{c} \beta \mathrm{c} \gamma\right] \text {, } \\
& h_{2}=m\left[y \dot{\alpha}^{2}-2 \dot{x} \dot{\alpha}\right]+m e\left[\left(\dot{\alpha}^{2}+\dot{\beta}^{2}+\dot{\gamma}^{2}\right) \mathrm{c} \beta \mathrm{s} \gamma+2 \dot{\alpha} \dot{\gamma} \mathrm{s} \gamma+2 \dot{\beta} \dot{\gamma} \mathrm{s} \beta \mathrm{c} \gamma\right] \text {, } \\
& h_{3}=m e\left[\dot{\beta}^{2} \mathrm{~s} \beta \mathrm{s} \gamma+\dot{\gamma}^{2} \mathrm{~s} \beta \mathrm{s} \gamma-2 \dot{\beta} \dot{\gamma} \mathrm{c} \beta \mathrm{c} \gamma\right] \text {, } \\
& h_{4}=-2 m[x \dot{x} \dot{\alpha}+y \dot{y} \dot{\alpha}] \\
& -m e\left[2 \dot{x} \dot{\alpha} \mathrm{c} \gamma+\left(2 \dot{y} \dot{\alpha}-x \dot{\beta}^{2}-x \dot{\gamma}^{2}\right) \mathrm{c} \beta \mathrm{s} \gamma\right. \\
& \left.-2 x \dot{\alpha} \dot{\gamma} \mathrm{s}-2 x \dot{\beta} \dot{\gamma} \mathrm{s} \beta \mathrm{c} \gamma+y \dot{\gamma}^{2} \mathrm{c} \gamma-2 y \dot{\alpha} \dot{\beta} \mathrm{s} \beta \mathrm{s} \gamma+2 y \dot{\alpha} \dot{\gamma} \mathrm{c} \beta \mathrm{c} \gamma\right] \\
& -(A-B)\left[\dot{\beta}^{2} \mathrm{c} \beta \mathrm{s} \gamma \mathrm{c} \gamma+2 \dot{\alpha} \dot{\gamma} \mathrm{s}^{2} \beta \mathrm{s} \gamma \mathrm{c} \gamma+\dot{\beta} \dot{\gamma} \mathrm{s} \beta\left(\mathrm{c}^{2} \gamma-\mathrm{s}^{2} \gamma\right)\right] \\
& -2\left(A \mathrm{~s}^{2} \gamma+B \mathrm{c}^{2} \gamma-C\right) \dot{\alpha} \dot{\beta} \mathrm{s} \beta \mathrm{c} \beta+C \dot{\beta} \dot{\gamma} \mathrm{s} \beta, \\
& h_{5}=m e\left[2 \dot{x} \dot{\alpha}-y \dot{\alpha}^{2}\right] \mathrm{s} \beta \mathrm{s} \gamma+(A-B)\left[2 \dot{\beta} \dot{\gamma} \mathrm{s} \gamma \mathrm{c} \gamma-\dot{\alpha} \dot{\gamma} \mathrm{s} \beta\left(\mathrm{c}^{2} \gamma-\mathrm{s}^{2} \gamma\right)\right] \\
& +\left(A \mathrm{~s}^{2} \gamma+B \mathrm{c}^{2} \gamma\right) \dot{\alpha}^{2} \mathrm{~s} \beta \mathrm{c} \beta-C\left[\dot{\alpha}^{2} \mathrm{~s} \beta \mathrm{c} \beta+\dot{\alpha} \dot{\gamma} \mathrm{s} \beta\right], \\
& h_{6}=-m e\left[2 \dot{y} \dot{\alpha}+x \dot{\alpha}^{2}\right] \mathrm{s} \gamma-m e\left[2 \dot{x} \dot{\alpha}-y \dot{\alpha}^{2}\right] \mathrm{c} \beta \mathrm{c} \gamma \\
& +(A-B)\left[\left(\dot{\alpha}^{2} \mathrm{~s}^{2} \beta-\dot{\beta}^{2}\right) \mathrm{s} \gamma \mathrm{c} \gamma+\dot{\alpha} \dot{\beta}\left(\mathrm{c}^{2} \gamma-\mathrm{s}^{2} \gamma\right) \mathrm{s} \beta\right]+C \dot{\alpha} \dot{\beta} \mathrm{s} \beta .
\end{aligned}
$$

For a shorter notation, the abbreviations $\mathrm{c} \gamma=\cos \gamma$ and $\mathrm{c}^{2} \gamma=\cos ^{2} \gamma$ are used. As the points $C_{1}$ and $S$ are not directly paramerterized, the rigid body formula ${ }_{R} \mathbf{v}_{P}={ }_{R} \mathbf{v}_{G}+{ }_{R} \boldsymbol{\Omega} \times{ }_{R} \mathbf{r}_{G P}$ is used to obtain the Jacobian ${ }_{R} \mathbf{J}_{P}={ }_{R} \mathbf{J}_{G}-{ }_{R} \tilde{\mathbf{r}}_{G P R} \mathbf{J}_{R}$ of an arbitrary body point $P$, where Jacobian ${ }_{R} \mathbf{J}_{R}=\mathbf{A}_{R B B} \mathbf{J}_{R}$ holds. The right hand side of Eq. (20) contains the generalized gravitational force

$$
\begin{aligned}
\mathbf{f}_{G} & =-m g_{R} \mathbf{J}_{S}{ }^{\mathrm{T}}{ }_{R} \mathbf{e}_{z}^{I}=-m g\left({ }_{R} \mathbf{J}_{G}-{ }_{R} \tilde{\mathbf{r}}_{G S R} \mathbf{J}_{R}\right)^{\mathrm{T}}{ }_{R} \mathbf{e}_{z}^{R} \\
& =-m g\left[\begin{array}{lllllll}
0 & 0 & 1 & 0 & e \mathrm{c} \beta \mathrm{s} \gamma & e \mathrm{~s} \beta \mathrm{c} \gamma
\end{array}\right]^{\mathrm{T}},
\end{aligned}
$$

the normal and tangential generalized contact forces $\mathbf{w}_{N} \lambda_{N}$ and $\mathbf{W}_{T} \boldsymbol{\lambda}_{T}$ with generalized force directions

$$
\begin{aligned}
& \mathbf{w}_{N}={ }_{R} \mathbf{J}_{C_{1}}{ }_{R}{ }_{R} \mathbf{e}_{z}^{R}=\left({ }_{R} \mathbf{J}_{G}-{ }_{R} \tilde{\mathbf{r}}_{G C_{1} R} \mathbf{J}_{R}\right)^{\mathrm{T}}{ }_{R} \mathbf{e}_{z}^{R} \\
& =\left[\begin{array}{llllll}
0 & 0 & 1 & 0 & -r \mathrm{c} \beta & 0
\end{array}\right]^{\mathrm{T}} \text {, } \\
& \mathbf{W}_{T}={ }_{R} \mathbf{J}_{C_{1}}{ }^{\mathrm{T}}\left[{ }_{R} \mathbf{e}_{x}^{R} \quad{ }_{R} \mathbf{e}_{y}^{R}\right]=\left({ }_{R} \mathbf{J}_{G}-{ }_{R} \tilde{\mathbf{r}}_{G C_{1} R} \mathbf{J}_{R}\right)^{\mathrm{T}}\left[{ }_{R} \mathbf{e}_{x}^{R} \quad{ }_{R} \mathbf{e}_{y}^{R}\right] \\
& =\left[\begin{array}{cccccc}
1 & 0 & 0 & r \mathrm{c} \beta-y & 0 & r \\
0 & 1 & 0 & x & -r \mathrm{~s} \beta & 0
\end{array}\right]^{\mathrm{T}}
\end{aligned}
$$

and writes as $\mathbf{f}^{\mathrm{ext}}=\mathbf{f}_{G}+\mathbf{w}_{N} \lambda_{N}+\mathbf{W}_{T} \boldsymbol{\lambda}_{T}$. The contact point $C_{1}$ does not detach during the inversion of the tippedisk, see [12]. This motivates the bilateral constraint $\mathbf{g}_{N}=0$, which forces the gap from Eq. (4) to zero. The application of the bilateral constraint on position level leads to a system of differential algebraic equations (DAE) with index three. This index can be reduced by formulating the bilateral constraint on acceleration level

$$
\text { for } t=0 \quad \mathbf{g}_{N}(0)=\dot{\mathbf{g}}_{N}(0)=0, \text { for } t \geq 0 \quad \ddot{\mathbf{g}}_{N}=\mathbf{w}_{N}^{\mathrm{T}} \ddot{\mathbf{q}}+\dot{\mathbf{w}}_{N}^{\mathrm{T}} \dot{\mathbf{q}}=0 .
$$

In this case the scalar normal force $\lambda_{N}$ takes the role of a Lagrange multiplier, which forces the constraint to be fulfilled. In [12] it is shown that smooth Coulomb friction

$$
\boldsymbol{\lambda}_{T}=\mu \lambda_{N} \frac{\boldsymbol{\gamma}_{T}}{\left\|\boldsymbol{\gamma}_{T}\right\|+\varepsilon}
$$


with the friction coefficient $\mu$ and the smoothing parameter $\varepsilon$ is sufficient to describe the inversion phenomenon qualitatively. The kinematic quantity $\gamma_{T}$ describes the relative slip velocity between the associated contact points $C_{1}$ and $D_{1}$ with respect to the rotating $R$-frame and is defined as

$$
\boldsymbol{\gamma}_{T}:=\left[\begin{array}{c}
\gamma_{T x} \\
\gamma_{T y}
\end{array}\right]=\left[\begin{array}{l}
{ }_{R} \mathbf{e}_{x}^{R} \cdot\left({ }_{R} \mathbf{J}_{C_{1}} \dot{\mathbf{q}}\right) \\
{ }_{R} \mathbf{e}_{y}^{R} \cdot\left({ }_{R} \mathbf{J}_{C_{1}} \dot{\mathbf{q}}\right)
\end{array}\right]
$$

Moreover, we mention that the friction force $\boldsymbol{\lambda}_{T}$ here depends linearly on the normal contact force $\lambda_{N}$ and therefore directly on the Lagrange multiplier $\lambda_{N}$ of the bilateral constraint. The smoothing is hereby motivated, as set-valued Coulomb-Contensou friction couples drilling and tangential friction [17]. If the macroscopic contact point experiences a spinning velocity, then from a microscopic point of view the associated contact area is forced to be in slip state. As the tippedisk is always spinning during inversion, the contact point $C_{1}$ slips permanently, such that smooth Coulomb friction is justified.

\section{SIMULATION}

Introducing the trivial kinematic relation $\dot{\mathbf{q}}=\mathbf{u}$, which is combined with the equation of motion from Eq. (20) and the bilateral constraint on acceleration level $\ddot{\mathbf{g}}_{N}(t)=0$, considering smooth coulomb friction, the system

$$
\underbrace{\left[\begin{array}{ccc}
\mathbf{I} & \mathbf{0} & \mathbf{0} \\
\mathbf{0} & \mathbf{M} & -\mathbf{W}_{N T} \\
\mathbf{0} & \mathbf{w}_{N}^{\mathrm{T}} & \mathbf{0}
\end{array}\right]}_{\mathbf{A}}\left[\begin{array}{c}
\dot{\mathbf{q}} \\
\dot{\mathbf{u}} \\
\lambda_{N}
\end{array}\right]=\left[\begin{array}{c}
\mathbf{u} \\
\mathbf{h}+\mathbf{f}_{G} \\
-\dot{\mathbf{w}}_{N}^{\mathrm{T}} \dot{\mathbf{q}}
\end{array}\right],
$$

with $\mathbf{W}_{N T}:=\mathbf{w}_{N}-\mu \mathbf{W}_{T} \frac{\boldsymbol{\gamma}_{T}}{\left\|\boldsymbol{\gamma}_{T}\right\|+\varepsilon}$ is obtained. Equation (41) corresponds to an linear equation system (with invertible A-matrix) such that it is possible to derive a first order ordinary differential equation (ODE) in generalized coordinates $\mathbf{q}$ and velocities $\mathbf{u}$. This ODE can be solved with any standard integrator, e.g., a four staged Runge-Kutta method. At this point we restrict us to the integrator ode $15 s$ from MATLAB. For a more detailed derivation, we refer to [12].

\subsection{Initial conditions and model parameters}

In this section, the results of numerical simulations using the derived minimal model are discussed. As shown in Figure 4, the tippedisk is called not inverted when $\beta=\frac{\pi}{2}$ and $\gamma=-\frac{\pi}{2}$ holds. In this configuration the center of gravity $S$ lies below the geometric center $G$. Vice versa, we call the disk inverted if $\beta=\frac{\pi}{2}$ and $\gamma=\frac{\pi}{2}$, i.e., the center of gravity $S$ lies above the geometric center $G$. Due to the periodicity of the trigonometric functions sine and cosine these definitions are not unique, since for $\beta=-\frac{\pi}{2}$ and $\gamma=\frac{\pi}{2}$ the tippedisk is also in a inverted configuration. However, the following numerical results show that $\beta$ is in the range of $(0, \pi)$ during the inversion, so this ambiguity does not matter.
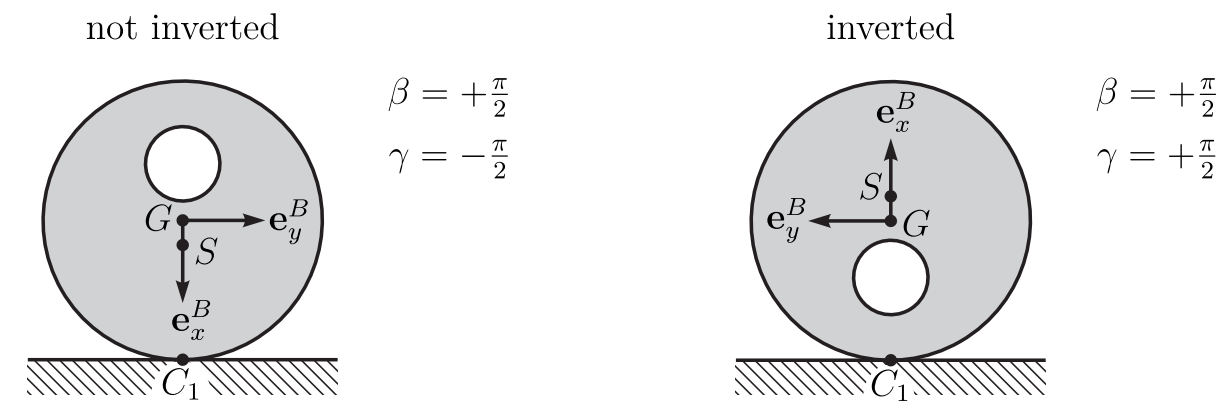

Figure 4: Non-inverted and inverted configuration of the tippedisk. 
The initial conditions, at time $t_{0}=0 \mathrm{~s}$, are given in Table 2 . The end time of the simulation is set to $t_{1}=5 \mathrm{~s}$. According to Table 2, the disk rotates initially with closed contact point in non-inverted orientation without slippage, i.e., $\left\|\boldsymbol{\gamma}_{T}\right\|=0$. For the following simulations, the friction coefficient $\mu=0.3$ and smoothing parameter $\varepsilon=0.1 \frac{\mathrm{m}}{\mathrm{s}}$ are chosen.

Table 2: Initial condition: Perturbed non-inverted spinning with closed contact.

\begin{tabular}{|c|c|c|c|c|c|}
\hline Coordinate & Magnitude & Unit & Velocity & Magnitude & Unit \\
\hline$x_{0}$ & 0 & $\mathrm{~m}$ & $\dot{x}_{0}$ & 0 & $\mathrm{~m} / \mathrm{s}$ \\
\hline$y_{0}$ & 0 & $\mathrm{~m}$ & $\dot{y}_{0}$ & 0 & $\mathrm{~m} / \mathrm{s}$ \\
\hline$z_{0}$ & 0.045 & $\mathrm{~m}$ & $\dot{z}_{0}$ & 0 & $\mathrm{~m} / \mathrm{s}$ \\
\hline$\alpha_{0}$ & 0 & $\mathrm{rad}$ & $\dot{\alpha}_{0}$ & 40 & $\mathrm{rad} / \mathrm{s}$ \\
\hline$\beta_{0}$ & $0.5 \pi$ & $\mathrm{rad}$ & $\dot{\beta}_{0}$ & 0 & $\mathrm{rad} / \mathrm{s}$ \\
\hline$\gamma_{0}$ & $-0.5 \pi+0.1$ & $\mathrm{rad}$ & $\dot{\gamma}_{0}$ & $-\dot{\alpha}_{0} \cos \left(\beta_{0}\right)=0$ & $\mathrm{rad} / \mathrm{s}$ \\
\hline
\end{tabular}

\subsection{Simulation results}

The numerical results of the system from Eq. (41), assuming smooth Coulomb friction Eq. (39) and initial conditions from Table 2 are shown in color in Figure 5. For comparison, the results of the quaternion-based model from [12], which assumes unilateral contact and smooth Coulomb friction, are shown in black (dotted/dashed). At $t=0 \mathrm{~s}$, the tippedisk is almost in the non-inverted configuration, as $\beta=+\frac{\pi}{2}$ and $\gamma=-\frac{\pi}{2}+0.1$ holds. Starting from this, the angle $\gamma$ increases quickly from $-\frac{\pi}{2}$ and ends in an asymptotic oscillation around $+\frac{\pi}{2}$. During this first stage of motion the inclination angle $\beta$ does only change slightly, such that the inversion of the tippedisk can be directly related to the change of the angle $\gamma$. Both angles $\beta$ and $\gamma$ are increasingly superimposed by small oscillations with higher frequency. In the $z_{S}$-graph, the height of the center of gravity $S$ is shown, growing from $r-e$ to $r+e$, which also indicates that the disk ends up in an inverted configuration with closed contact. During this inversion process, the kinetic energy $E_{\text {kin }}$ decreases while the potential energy $E_{\text {pot }}$ increases, such that the total energy $E_{\text {tot }}$ dissipates from $0.38 \mathrm{Nm}$ to $0.36 \mathrm{Nm}$ for $t<0.5 \mathrm{~s}$. After this initial fast decay, the total energy slowly decreases as the tippedisk rotates near the inverted stationary solution. In the considered time interval, the rotation angle $\alpha$ increases almost linearly. The associated spinning velocity $\dot{\alpha}$ initially increases as the height $z_{S}$ of the center of gravity drops, followed by a saturating decrease on an 'intermediate' timescale. The long-term behavior is characterized by a slow decrease of the spinning velocity $\dot{\alpha}$. 

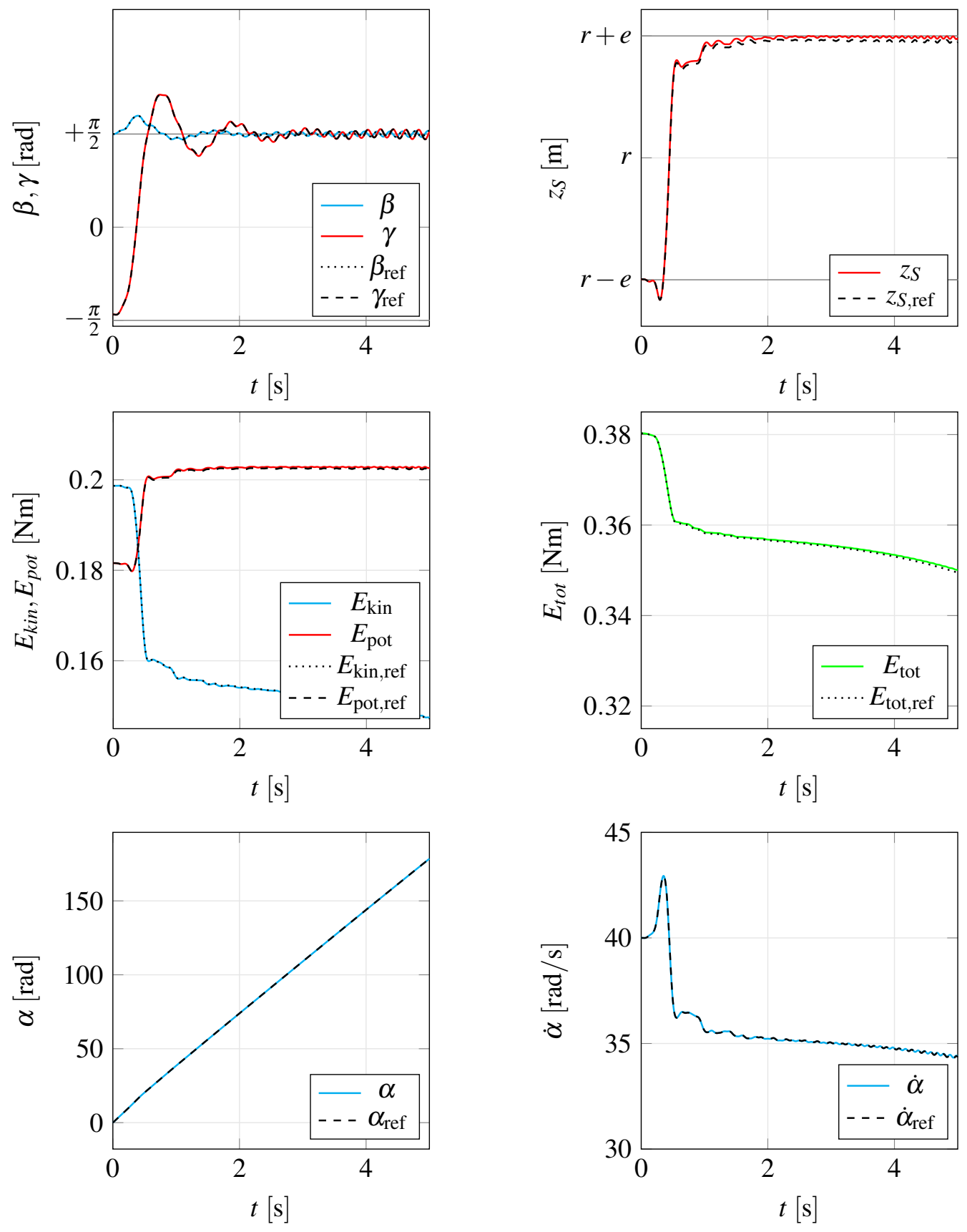

Figure 5: Simulation results showing the inversion of the tippedisk. The corresponding initial conditions are given in Table 2.

\section{DISCUSSION}

The simulation results from Fig. 5 show that the derived model with smooth Coulomb friction and a bilateral constrained contact point is able to describe the inversion behavior of the tippedisk. Comparison with the quaternion-based time stepping model proves that both models lead to the same results (with respect to numerical error), which shows the correctness of the new minimal model for the inversion phenomenon. As the Change of the spinning velocity $\dot{\alpha}$ is relatively small, the associated rotation angle $\alpha$ depends almost linearly on time $t$, i.e., $\alpha$ is approximately given as $\alpha(t) \approx \Omega t$ with constant spinning velocity $\Omega$. At this point, the derivation of the new minimal model seems artificial, since other models from [12] already describe the inversion phenomenon of the tippedisk correctly and even lead to the exactly same results, cf. Fig. 5. However, to characterize the stability of the inverted and non-inverted stationary solutions, it is convenient 
to linearize the equations of motion around $\beta=+\frac{\pi}{2}$ and $\gamma= \pm \frac{\pi}{2}$. A given parametrization of the geometric center $G$ (or center of gravity $S$ ) with respect to the inertial frame $I$ yields system equations that depend explicitly on the angle $\alpha$, see [12]. As $\alpha(t)$ is a function in time $t$, the linearized system matrices are time dependent, therefore Lypunov's indirect method can not be applied and Floquet theory must be used, which greatly complicates the closed form analysis. In this work, we have introduced a new parametrization of the geometric center $G$ with respect to the rotating $R$-frame, which led to system equations that do not explicitly depend on $\alpha$ and thus do not depend on time $t$. This has the advantage that the linearized system matrices are constant and thus Lyapunov's indirect method can be applied to study the local stability behavior.

\section{CONCLUSIONS}

The tippedisk introduced in [12] serves as a link between analytical mechanics, theoretical mechanics and nonlinear dynamics. Our aim is to understand the nonlinear behavior behind the inversion phenomenon of the tippedisk. Therefore, a mathematical description is sought that will form the basis for future stability analyses of the nonlinear system. Depending on the parametrization, the system equations vary in their suitability for dynamic analysis. In this work, we have introduced a new minimal model of the tippedisk, which qualitatively describes the inversion phenomenon and has some advantages for future dynamical considerations, such as constant system matrices for the linearization around the inverted and non-inverted configurations. Based on the parametrization presented here, [13] applies Lyapunov's indirect method to obtain a closed-form expression for the critical spinning velocity $\Omega_{\text {crit }}$ above which the inverted spinning solution becomes stable. Moreover, the linear stability analysis indicates different timescales, suggesting slow-fast dynamical behavior. By analyzing this singularly perturbed structure, the complexity and order of the model can be reduced to obtain a lower dimensional dynamical system that describes the inversion phenomenon of the tippedisk, cf. [13, 14].

\section{ACKNOWLEDGMENTS}

The authors declare that they have no conflicts of interest. This research received no specific grant from any funding agency in the public, commercial, or non-profit sectors.

\section{REFERENCES}

[1] Moffatt, H.K.: Euler's disk and its finite-time singularity. Nature 404(6780) (2000) 833-834

[2] Leine, R.I.: Experimental and theoretical investigation of the energy dissipation of a rolling disk during its final stage of motion. Arch Appl Mech 79(11) (2009) 1063-1082

[3] Garcia, A., Hubbard, M.: Spin reversal of the rattleback: theory and experiment. Proc. R. Soc. Lond. A 418(1854) (1988) 165-197

[4] Borisov, A.V., Mamaev, I.S.: Strange attractors in rattleback dynamics. Phys.-Usp. 46(4) (2003) 393

[5] Magnus, K.: Kreisel. Springer, Berlin, Heidelberg, New York (1971)

[6] Cohen, R.J.: The tippe top revisited. American Journal of Physics 45(1) (1977) 12-17

[7] Bou-Rabee, N.M., Marsden, J.E., Romero, L.A.: Tippe Top Inversion as a DissipationInduced Instability. SIAM J. Appl. Dyn. Syst. 3(3) (2004) 352-377

[8] Rauch-Wojciechowski, S.: What does it mean to explain the rising of the tippe top? Regul. Chaot. Dyn. 13(4) (2008) 316-331

[9] Moffatt, K., Shimomura, Y.: Spinning eggs — a paradox resolved. Nature 416(6879) (2002) $385-386$ 
[10] Branicki, M., Shimomura, Y.: Dynamics of an axisymmetric body spinning on a horizontal surface. IV. Stability of steady spin states and the 'rising egg' phenomenon for convex axisymmetric bodies. Proc. R. Soc. A. 462(2075) (2006) 3253-3275

[11] Cross, R.: Why does a spinning egg rise? European Journal of Physics 39(2) (2018) 025002

[12] Sailer, S., Eugster, S.R., Leine, R.I.: The Tippedisk: a Tippetop Without Rotational Symmetry. Regul. Chaot. Dyn. 25(6) (2020) 553-580

[13] Sailer, S., Leine, R.I.: Model reduction of the tippedisk: a path to the full analysis. Nonlinear Dyn 105(3) (2021) 1955-1975

[14] Sailer, S., Leine, R.I.: Singularly perturbed dynamics of the tippedisk. arxiv:2109. 02542 [physics.class-ph] (2021)

[15] Le Saux, C., Leine, R.I., Glocker, C.: Dynamics of a Rolling Disk in the Presence of Dry Friction. J Nonlinear Sci 15(1) (2005) 27-61

[16] Glocker, C.: Set-valued Force Laws: Dynamics of Non-smooth Systems. Volume 1 of Lecture Notes in Applied Mechanics. Springer Science \& Business Media (2001)

[17] Leine, R.I., Glocker, C.: A set-valued force law for spatial Coulomb-Contensou friction. European Journal of Mechanics-A/Solids 22(2) (2003) 193-216 